TRANSACTIONS OF THE

AMERICAN MATHEMATICAL SOCIETY

Volume 354, Number 6, Pages 2399-2416

S 0002-9947(02)02892-1

Article electronically published on February 14, 2002

\title{
THE ONE PHASE FREE BOUNDARY PROBLEM FOR THE $p$-LAPLACIAN WITH NON-CONSTANT BERNOULLI BOUNDARY CONDITION
}

\author{
ANTOINE HENROT AND HENRIK SHAHGHOLIAN
}

\begin{abstract}
Our objective, here, is to generalize our earlier results on the existence of classical convex solution to a free boundary problem with a Bernoullitype boundary gradient condition and with the $p$-Laplacian as the governing operator. The main theorems of this paper assert that the exterior and the interior free boundary problem with a Bernoulli law, i.e. with a prescribed pressure $a(x)$ on the "free" streamline of the flow, have convex solutions provided the initial domains are convex. The continuous function $a(x)$ is subject to certain convexity properties. In our earlier results we have considered the case of constant $a(x)$. In the lines of the proof of the main results we also prove the semi-continuity (up to the boundary) of the gradient of the $p$-capacitary potentials in convex rings, with $C^{1}$ boundaries.
\end{abstract}

\section{INTRODUCTION}

Nonlinear potential flows, of power-law types, past convex profiles are the main object of investigation in this paper. The problem arises when a fluid flows in porous medium around an obstacle. In certain industrial problems such as shape optimization, painting, and galvanization, one seeks to find level lines (surfaces in higher dimension) of the potential function (i.e., streamlines) with prescribed pressure on it. The latter is given by Bernoulli's law

$$
|\nabla u|=\text { prescribed on the free streamline, }
$$

where $u$ is the potential function which corresponds to the flow vector $-\gamma \nabla u$; here $-\gamma$ is the conductivity of the flow.

A more general, and maybe realistic, situation is the case of power-law flows

$$
-\gamma|\nabla u|^{p-2} \nabla u, \quad(1<p<\infty) .
$$

To describe the problem mathematically let $K$ be a convex domain in $\mathbb{R}^{n}(n \geq 2)$ and $a(x)$ be a continuous function. Then we seek to find another convex domain

Received by the editors July 14, 2000 and, in revised form, August 16, 2001.

1991 Mathematics Subject Classification. Primary 35R35, 35J70, 76S05.

Key words and phrases. Free boundary, convexity, non-linear joining conditions.

The first author thanks Göran Gustafsson Foundation for several visiting appointments to RIT in Stockholm.

The second author was partially supported by the Swedish Natural Science Research Council and STINT. He also thanks Institute Elie Cartan for their hospitality. Both authors thank A. Petrosyan for some crucial remarks. 
$\Omega$ containing the closure of $K$ (this is called the exterior problem) such that the $p$-capacitary potential $u$ of $\Omega \backslash \bar{K}$ satisfies the Bernoulli boundary condition

$$
\lim _{x \rightarrow y}|\nabla u(x)|=a(y), \quad x \in \Omega, \quad y \in \partial \Omega .
$$

Similarly one may ask for an interior domain (the interior problem) $\Omega \subset \bar{\Omega} \subset K$ such that (1.1) holds on $\partial \Omega$, with $x \in K \backslash \bar{\Omega}$.

We recall that the $p$-capacitary potential $u$ of a ring-shaped region $D_{2} \backslash \overline{D_{1}}$, where $D_{1}$ and $D_{2}$ are two nested open domains $\left(\bar{D}_{1} \subset D_{2}\right)$, is the solution of

$$
\begin{cases}\Delta_{p} u=0 & \text { in } D_{2} \backslash \overline{D_{1}}, \\ u=1 & \text { on } \partial D_{1}, \\ u=0 & \text { on } \partial D_{2} .\end{cases}
$$

Here $\Delta_{p}$, for $1<p<\infty$, denotes the $p$-Laplace operator

$$
\Delta_{p} u:=\operatorname{div}\left(|\nabla u|^{p-2} \nabla u\right) .
$$

This is to be understood in a weak sense, see below. The case $p=2$ is the ordinary Laplacian. The general case is an important prototype of degenerate elliptic operators. We also remark that we may abuse the terminology by also calling the function $(1-u)$ for the $p$-capacitary potential.

Because of its importance, the flow problem described above, for $p=2$, has gained a lot of attention in the past 20 years, both numerically and analytically (see FR] for a good account of backgrounds, overview and references of the subject). However, until recently the case of general $p$ has been out of reach due to the lack of smoothness of the operator $\Delta_{p}$. We refer the author to [HKM] for background about such degenerate operators. Cf. also [Di] for the parabolic case.

It is noteworthy that the technical parts of the proofs of the so far existing results for the case $p=2$ rely heavily on, by now classical, papers such as AC] and $[\mathrm{CS}]$; see e.g. $[\mathrm{A}]$ and the references therein. The core difficulty of the problem is the $C^{1}$-regularity of the boundary and the semi-continuity of the gradient up to the boundary, see Theorem 1.3 below. Yet another difficulty that appears in such problems is to show the non-vanishing of the gradient of the $p$-capacitary potential on the boundary. This is called non-degeneracy of the solution and it enters into the proof (all proofs the authors know) in a crucial way. These problems are tackled for the first time in this paper, and we believe they constitute, besides the old ideas of the supersolutions technique of A. Beurling, the operator method of A. Acker, and the use of Nash-Moser inverse function theorem by R. Hamilton, the main technical ingredients in any proof for the existence of classical solutions. We use the term classical since it is known that $p$-capacitary potentials in convex rings are real analytic functions inside the ring (see $[\mathrm{L}]$ ). However, the term "classical" in this paper refers to (1.1).

At this stage we want to refer the reader to the previous papers by the authors [HS1]- HS3] and the references therein.

Our main result in this paper is a generalization of [HS2]-[HS3] to the case of the non-constant Bernoulli boundary condition

$$
|\nabla u|=a(x) \quad \text { on } \partial\{u>0\}
$$

with $a(x)$ a positive continuous function such that $1 / a(x)$ is locally concave on $\mathbb{R}^{n} \backslash K$ for the exterior problem. The latter means that $1 / a$ is concave on each line segment in the set $\mathbb{R}^{n} \backslash K$. The same generalization is made for the interior 
problem where $1 / a(x)$ is assumed to be convex on $K$. Obviously we need also to assume that $a(x)$ stays away from zero, since otherwise there may be no solutions at all. The latter can easily be checked for spherical solutions. In general we need to have a "supersolution" for the given function $a(x)$, in order to start a Perron method; see the proof of Theorems 1.1-1.2. Now let us define two classes of test functions.

Definition 1.1 (Exterior). Let $D$ be a bounded domain in $\mathbb{R}^{n}$, and $a(x)$ a continuous function. Define $\mathcal{E}=\mathcal{E}(D, a(x))$ to be the class of all continuous functions $v$ on $\mathbb{R}^{n}$ such that:

1) $v \in C^{0,1}\left(\mathbb{R}^{n} \backslash \bar{D}\right)$;

2) $v=1$ on $\partial D$;

3) $\Delta_{p} v \leq 0$ in $\{v>0\} \backslash \bar{D}$

4) $v$ has compact support;

5) $|\nabla v|(x) \leq a(x)$ on $\partial\{v>0\}$.

The set $\{v>0\}$ will be denoted by $\Omega=\Omega(v)$. Similarly we define the "interior" class, $\mathcal{I}$.

Definition 1.2 (Interior). Let $D$ be a bounded domain in $\mathbb{R}^{n}$, and $a(x)$ a continuous function. Define $\mathcal{I}=\mathcal{I}(D, a(x))$ to be the class of all continuous functions $v$ on $\mathbb{R}^{n}$ such that:

1) $v \in C^{0,1}(D)$;

2) $v=1$ on $\mathbb{R}^{n} \backslash D$;

3) $\Delta_{p} v \leq 0$ in $\{v>0\} \cap D$;

4) the interior of $\{v \equiv 0\}$ is non-void;

5) $|\nabla v|(x) \leq a(x)$ on $\partial\{v>0\} \cap D$.

For the interior case we let the set $\{v=0\}$ be denoted by $\Omega=\Omega(v)$. The dependence of $\mathcal{E}$ and $\mathcal{I}$ on $D$ and $a(x)$ will be suppressed if there is no ambiguity. The requirement 3 ) in these definitions is to be understood in the weak sense, i.e.,

$$
0 \leq \int|\nabla v|^{p-2} \nabla v \cdot \nabla \psi
$$

for all $0 \leq \psi \in C_{o}^{\infty}(\{v>0\} \backslash\{u \equiv 1\})$.

Since the class $\mathcal{E}$ and $\mathcal{I}$ only contain Lipschitz functions, 5) in definitions 1.1-1.2 also needs attention. The best way to interpret 5 ) is to require $u$ to satisfy the following condition. For $\delta>0$ there exists a small neighborhood $U_{\delta}$ of $\partial \Omega$ such that

$$
\sup _{x \in U_{\delta} \cap \Omega} \lim _{\epsilon \rightarrow 0} \frac{|u(x+\epsilon e)-u(x)|}{\epsilon} \leq a(x)+\delta,
$$

for all directions $e$.

Theorem 1.1 (Exterior). Let $K$ be a convex domain, and $a(x)$ a continuous function with $0<c_{0} \leq a(x) \leq C_{0}$ on $\mathbb{R}^{n}$. Suppose moreover $1 / a(x)$ is locally concave on $\mathbb{R}^{n} \backslash K$. Then there exists a convex $C^{1}$ domain $\Omega$ such that the p-capacitary potential $u$ of $\Omega \supset \bar{K}$ is a classical solution to the exterior Bernoulli free boundary problem. Moreover, if $K$ is bounded and for some $x^{0} \in K$,

$$
t: \rightarrow \operatorname{ta}\left(t\left(x-x^{0}\right)+x^{0}\right)
$$

is increasing for all $x \in \mathbb{R}^{n}$, then $\Omega$ is unique. 
In a recent work MPS J. Manfredi, A. Petrosyan and H. Shahgholian have considered the case of $p=+\infty$ for Theorem 1.1. The analysis in [MPS] is based on the existence of classical solution for $1<p<\infty$, i.e., Theorem 1.1 above. The core difficulty that appears in [MPS] is that as $p \rightarrow \infty$, the solutions have a tendency of becoming irregular and the uniformity in the $C^{1}$ norm is lost. See [MPS] for more details.

Theorem 1.2 (Interior). Let $K$ be a convex domain, and $a(x)$ a continuous function in $K$, with $0<c_{0} \leq a(x) \leq C_{0}$. Suppose moreover $\mathcal{I}(K, a)$ is non-empty and that $1 / a(x)$ is a convex function on $K$. Then there exists a (not necessarily unique) convex domain $\Omega \subset \bar{\Omega} \subset K$, with $C^{1}$ boundary such that the p-capacitary potential $u$ of $K \backslash \bar{\Omega}$ is a classical solution to the interior Bernoulli free boundary problem.

The same technique, used by [MPS], for the exterior problem as $p \rightarrow \infty$ appears to work in the case of generalization of Theorem 1.2 to $p=\infty$. However, the "small" technical details to be filled out are yet to be handled.

The regularity of the free boundary in both theorems depends strongly on the regularity of the function $a(x)$, and it is in general a hard problem. We refer to the paper of A. Vogel for some details for the case of constant $a(x)$. The regularity of the free boundary, in the general case of non-constant $a(x)$, remains yet to be studied.

Finally let us formulate a technical result, which, besides being the main technical ingredient in this paper, is of more general interest in partial differential equations.

Theorem 1.3. Let $D_{1}$ and $D_{2}$ be two nested open convex domains $\left(D_{1} \subset D_{2}\right)$ and denote by $u$ the p-capacitary potential of $D_{2} \backslash D_{1}$. Suppose also that $\partial D_{1}$ and $\partial D_{2}$ are $C^{1}$. Then $|\nabla u|$ is semi-continuous in $\overline{D_{2} \backslash D_{1}}$, and non-tangentially continuous up to $\partial D_{1} \cup \partial D_{2}$.

Since Theorem 1.3 is of a rather technical character, we prove it in the next section, before the proofs of the main results.

\section{Technichl Lemmas And the Proof of Theorem 1.3}

In this section we will first introduce some basic definitions that will be used for proving the theorems. We will also prove the main technical difficulties that arise in the case of non-constant $a(x)$.

Definition 2.1 (Extremal points). For a bounded domain $D \in \mathbb{R}^{n}$, a point $x \in$ $\partial D$ is said to be extremal if there exists a supporting plane to $D$ touching $\partial D$ at $x$ only. We denote the set of all extremal points of $D$ by $E_{D}$.

Remark 2.1. By Krein-Milman's theorem

$$
\text { convex } \operatorname{hull}(D)=\text { convex } \operatorname{hull}\left(\overline{E_{D}}\right) \text {. }
$$

Lemma 2.2 (Exterior barrier). Let $D$ be a convex domain in $\mathbb{R}^{n}$ and suppose $u$ is a continuous nonnegative function on $B\left(x^{0}, r\right)$, p-harmonic in $B\left(x^{0}, r\right) \cap D$, with $x^{0} \in \partial D$. Let also $u=0$ on $\partial D$. If $\partial D$ is not $C^{1}$ at $x^{0}$, i.e., $D$ has (at least) two supporting planes at $x^{0}$, then

$$
\lim _{x \rightarrow x^{0}}|\nabla u(x)|=0, \quad x \in D
$$


Lemma 2.3 (Interior barrier). Let $D$ be a convex domain in $\mathbb{R}^{n}$ and suppose $u$ is a continuous nonnegative function on $B\left(x^{0}, r\right)$, p-harmonic in $B\left(x^{0}, r\right) \backslash D$, with $x^{0} \in \partial D$. Let also $u=0$ on $\partial D$. If $\partial D$ is not $C^{1}$ at $x^{0}$, i.e., $D$ has (at least) two supporting planes at $x^{0}$, then

$$
\lim _{x \rightarrow x^{0}}|\nabla u(x)|=\infty, \quad x \in B\left(x^{0}, r\right) \backslash D .
$$

The proof of these lemmas follow from standard theory using barriers on conical boundary points. The existence of such barriers are proven in [Do, see also $\mathrm{K}$ ].

Definition 2.2 (Blow-up). Let the function $u$ be defined in $B\left(x^{0}, 1\right)$. Then we define the scaled function $u_{r}(x)$ in $B(0,1)$ by

$$
u_{r}(x)=\frac{u\left(r x+x^{0}\right)-u\left(x^{0}\right)}{r} .
$$

Suppose now that $u$ is Lipschitz in $B\left(x^{0}, 1\right)$. Then $u_{r}$ is uniformly Lipschitz. Thus for any sequence $\left\{r_{j}\right\} \searrow 0$, there exists a subsequence (again labeled $r_{j}$ ) such that $u_{r_{j}}$ converges locally in $C^{\alpha}\left(\mathbb{R}^{n}\right)$ to a function $u_{0}$. Moreover if $u$ is $p$-harmonic in $\{u>0\}$ then $u_{0}$ is $p$-harmonic in $\left\{u_{0}>0\right\}$ and $u_{0}(0)=0$.

Lemma 2.4. Let $u$ be the $p$-capacitary potential of an annular domain $D=D_{2} \backslash D_{1}$ with convex $C^{1}$ boundaries. Suppose moreover the gradient of u satisfies

$$
|\nabla u| \leq \Lambda_{0}<\infty
$$

uniformly in the region $D$. Then any convergence blow-up of $u_{r_{j}}$ at any boundary point gives a linear function $u_{0}=\alpha x_{1}^{+}$, after suitable rotation and translation. In particular, for any boundary point $x^{0}$

$$
u(x)=u\left(x^{0}\right)+\alpha\left(x_{1}-x_{1}^{0}\right)^{+}+o\left(r_{j}\right),
$$

in some rotated system. Here $o\left(r_{j}\right)$ depends on $x^{0}$.

Proof. The case $x^{0} \in \partial D_{1}$ was treated in [HS3 Lemma 2.7]. Let us suppose $x^{0} \in \partial D_{2}$. We scale $u$ at $x^{0}$ with the sequence $\left\{r_{j}\right\}$, where $r_{j} \searrow 0$; i.e.,

$$
u_{r_{j}}(x)=\frac{u\left(r_{j} x+x^{0}\right)}{r_{j}} .
$$

Recall J. Lewis' result $\left[\mathrm{L}\right.$ about convexity of the level sets of $u$, where $D_{1}$ and $D_{2}$ are convex. In particular the level sets of $u_{r_{j}}$ are convex. Next, by Lipschitz regularity, the functions $u_{r_{j}}$ will be uniformly Lipschitz in $B\left(0,1 / r_{j}\right)$. Hence for a subsequence they converge in $C_{l o c}^{\alpha}\left(\mathbb{R}^{n}\right)$ to a limit function $u_{0}$.

Now if $u_{0} \equiv 0$ then we are done. So suppose $u_{0} \not \equiv 0$. In this case one readily verifies that after suitable rotation

$$
\Delta_{p} u_{0}=0 \quad \text { in }\left\{x_{1}>0\right\}=: \Omega_{0}, \quad u_{0}=0 \text { on }\left\{x_{1}=0\right\}, \quad \text { and }\left|\nabla u_{0}\right| \leq \Lambda_{0} .
$$

By convexity of the level sets of $u$ we also have $\left\{u_{0}>t\right\}$ is convex for all $t \geq 0$. Finally one verifies that

Now define

$$
\sup _{x_{1}<2^{k}} u_{0}(x) \leq \Lambda_{0} 2^{k} .
$$

$$
C_{k}:=2^{-k} \sup _{x_{1}<2^{k}} u_{0},
$$

which is bounded according to the above estimate, and consider two cases:

Case 1: $C_{k} \geq c>0$, for all $k$; 
Case 2: $C_{k_{j}} \rightarrow 0$, for some subsequence $k_{j}$.

In Case 1 we show that the level sets are hyperplanes parallel to $\left\{x_{1}=0\right\}$. Then the function $u_{0}$ will be one dimensional and one can see (by direct computation) that the one dimensional solution is to be the linear function. Indeed, one has to use that $u^{\prime}>0$, which in turn is a result of the convexity of the level sets.

Next take an arbitrary level set $\mathcal{L}_{t}=\left\{u_{0}>t\right\}$ and suppose that $\partial \mathcal{L}_{t}$ is not a hyperplane parallel to $\left\{x_{1}=0\right\}$. By convexity of the level sets we may take $x^{1} \in$ $\partial \mathcal{L}_{t}$, with $e \perp \partial \mathcal{L}_{t}$ at $x^{1}$, and such that the supporting plane $\Pi=\left\{\left(x-x^{1}\right) \cdot e=0\right\}$ of $\mathcal{L}_{t}$ at $x^{1}$ has the following properties

$$
\mathcal{L}_{t} \subset \Pi_{+}:=\left\{\left(x-x^{1}\right) \cdot e>0\right\}
$$

and

$$
u_{0}(x) \leq u_{0}\left(x^{1}\right) \quad \text { in } \Pi_{-}:=\left\{\left(x-x^{1}\right) \cdot e<0\right\} .
$$

Now we want to perform a second blow-up of $u$ at $\infty$, i.e., a first blow-up of $u_{0}$ at the infinity point. Hence we define

$$
\left(u_{0}\right)_{R}=\frac{u_{0}\left(R x+y^{1}\right)}{R},
$$

where $y^{1}$ is any fixed point on $\Pi \cap\left\{x_{1}=0\right\}$. Then once again by the compactness argument $\left(\left|\nabla\left(u_{0}\right)_{R}\right| \leq \Lambda_{0}\right)$ we have a convergence subsequence $\left(u_{0}\right)_{R_{j}}$ converging to a limit function $u_{0 \infty}$ locally in the entire space $\mathbb{R}^{n}$, with the following properties:

$$
\begin{gathered}
u_{0 \infty} \not \equiv 0, \quad\left(\text { since } C_{k} \geq c>0\right), \\
\Delta_{p} u_{0 \infty}=0 \quad \text { in }\left\{x_{1}>0\right\} \quad \text { obvious, } \\
u_{0 \infty} \equiv 0 \quad \text { in }\{x \cdot e \leq 0\} .
\end{gathered}
$$

The latter depends on the fact that by (2.1) and the convexity of level sets we have

$$
\frac{u_{0}\left(R x+y^{1}\right)}{R} \leq \frac{u_{0}\left(x^{1}\right)}{R} \rightarrow 0 \quad \text { for } x \in\{x \cdot e \leq 0\}=\Pi_{-}-y^{1} .
$$

Now $e$ is not parallel to the $x_{1}$-axis, so that the cone $K^{\prime}=\left\{x_{1}>0\right\} \cap\{x \cdot e<0\}$ is non-void. Since $u_{0 \infty}$ is nonnegative $p$-harmonic in the domain $\left\{x_{1}>0\right\}$ and it is zero in $K^{\prime} \subset\left\{x_{1}>0\right\}$, it follows by the minimum principle that $u_{0 \infty} \equiv 0$. This contradicts (2.1).

For Case 2 we apply the comparison principle in the region $\left\{x_{1}<2^{k_{j}}\right\}$. Indeed, $u_{0} \leq C_{k_{j}} x_{1}$ on $\left\{x_{1}=0\right\} \cup\left\{x_{1}=2^{k_{j}}\right\}$. Using the comparison principle in the strip $\left\{0<x_{1}<2^{k_{j}}\right\}$ (see [GT, Theorem 3.7], the same proof works for the $p$-Laplacian) we'll have $u_{0} \leq C_{k_{j}} x_{1}$ in $\left\{x_{1}<2^{k_{j}}\right\}$. As $k_{j} \rightarrow \infty$ we obtain $u_{0}(x)=0$ for any $x \in \mathbb{R}^{n}$. Hence again $u(x)=o\left(r_{j}\right)$ with $\alpha=0$ and the proof is completed.

Lemma 2.5. Let $u$ be a solution to $\Delta_{p} u=0$ in a domain $\Omega$, and introduce the linear elliptic operator $L_{u}$ defined everywhere, except at critical points of $u, b y$

$$
L_{u}:=|\nabla u|^{p-2} \Delta+(p-2)|\nabla u|^{p-4} \sum_{k, l=1}^{n} \frac{\partial u}{\partial x_{k}} \frac{\partial u}{\partial x_{l}} \frac{\partial^{2}}{\partial x_{k} \partial x_{l}} .
$$

Then $L_{u}\left(|\nabla u|^{p}\right) \geq 0$ in $\Omega \backslash\{|\nabla u|=0\}$.

For a proof see [PP1], PP2 and the discussion in [HS2]. Observe that $L_{u}$ is uniformly elliptic in $\{u>0\} \backslash\left\{\lambda_{0} \leq|\nabla u| \leq \Lambda_{0}\right\}$, with $C^{\alpha}$ coefficients.

For two nested convex sets $D_{1} \subset D_{2}$, and for $x \in \partial D_{1}$ we denote by $T_{x, a}$ the supporting hyperplane at $x$ with the normal $a$ pointing away from $D_{1}$. Obviously, 
$T_{x, a}$ is not necessarily unique, depending on the geometry of $\partial D_{1}$. Now for each $x \in \partial D_{1}$ there corresponds a point $y_{x}$ (not necessarily unique) on $\partial D_{2} \cap\{z$ : $a \cdot(z-x)>0\}$ and such that $a \cdot\left(y_{x}-x\right)=\max a \cdot(z-x)$, where the maximum has been taken over all $z \in \partial D_{2} \cap\{z: a \cdot(z-x)>0\}$.

Lemma 2.6. Let $D_{1}$ and $D_{2}$ be two nested open convex domains $\left(D_{1} \subset D_{2}\right)$ and denote by $u$ the $p$-capacitary potential of $D_{2} \backslash D_{1}$. Then

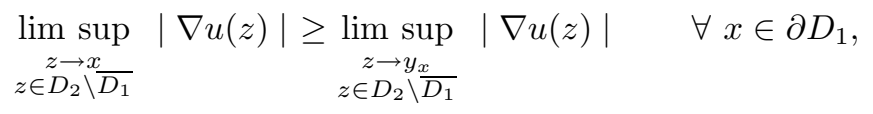

where $y_{x}$ is the point indicated in the discussion preceding this lemma.

For a proof of this lemma see [HS2]- $\mathrm{HS} 3$. Our next lemma is an infinitesimal version of Lemma 2.6.

Lemma 2.7. Retain the hypothesis in Lemma 2.6 and suppose also that $\partial D_{1}$ and $\partial D_{2}$ are $C^{1}$. Let $z \in D_{2} \backslash \bar{D}_{1}$ with $u(z)=s \in(0,1)$. Define a curve $\gamma_{z}(t)$ by

$$
\frac{d}{d t} \gamma_{z}(t)=\frac{\nabla u\left(\gamma_{z}(t)\right)}{\left|\nabla u\left(\gamma_{z}(t)\right)\right|^{2}}, \quad \gamma_{z}(s)=z
$$

Then $\gamma(t)$ is defined on $(0,1), u\left(\gamma_{z}(t)\right)=t$, and

$$
\left|\nabla u\left(\gamma_{z}(t)\right)\right| \nearrow \quad \text { as } t \nearrow \text {. }
$$

For a proof see MPS Lemma 5.1]. Cf. also [V]. The proof uses representation of the $p$-Laplacian in level sets coordinates, and the fact that the level sets are convex.

Using the technical lemmas above we can prove Theorem 1.3, which in turn is needed for the proof of the main theorems in this paper.

Proof of Theorem 1.3. Let us first show that

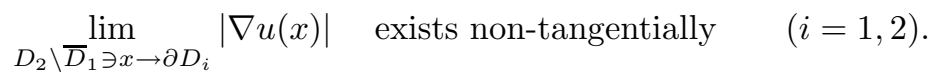

Non-tangentially here means that if we fix any cone of opening $\pi / 2-\epsilon$ with vertex at $x^{0}$ on the boundary of $\Omega$ and with the normal vector to the boundary at $x^{0}$, pointing inwards $\Omega$ as the axis of the cone, then the limit of the gradient exists if we approach the boundary point $x^{0}$ from this cone.

Now suppose (2.2) fails. Let us take a point $y \in \partial D_{i}$ where $(2.2)$ fails. We suppose $y$ is the origin and that the interior normal to $D_{i}$ at the origin (i.e. $y$ ) is $e_{1}$ (the first coordinate axis). Now the failure of (2.2) implies that there exists $z^{j} \rightarrow 0$ and $x^{j} \rightarrow 0$ such that

$$
\left|\nabla u\left(z^{j}\right)\right| \rightarrow \alpha_{1}, \quad\left|\nabla u\left(x^{j}\right)\right| \rightarrow \alpha_{2},
$$

with $\alpha_{1} \geq \alpha_{2}+\epsilon_{0}$ for some $\epsilon_{0}>0$. Moreover by the non-tangentiality

$$
\operatorname{dist}\left(z^{j}, \partial \Omega\right) \geq c_{0}\left|z^{j}\right|, \quad \operatorname{dist}\left(x^{j}, \partial \Omega\right) \geq c_{0}\left|x^{j}\right|
$$

for some $c_{0}>0$.

Let us define

$$
\begin{gathered}
S_{z^{j}}=\left\{x: u(x)=u\left(z^{j}\right)\right\}, \quad S_{x^{j}}=\left\{x: u(x)=u\left(x^{j}\right)\right\} \\
r_{j}=\left|z^{j}\right|, \quad t_{j}=\left|x^{j}\right| .
\end{gathered}
$$


We may also rearrange $z^{j}$ and $x^{j}$ such that $t_{j}>r_{j}$. Now by Lemma 2.4 for a subsequence and locally in $\mathbb{R}^{n}$

$$
u_{r_{j}}(x) \rightarrow u_{1}(x):=\alpha_{1} x_{1}^{+}, \quad u_{t_{j}}(x) \rightarrow u_{2}(x):=\alpha_{2} x_{1}^{+} .
$$

Let us define, accordingly, the scaled versions of the sets $S_{z^{j}}$ and $S_{x^{j}}$ by

$$
\tilde{S}_{z^{j}}=\left\{x: u_{r_{j}}(x)=u_{r_{j}}\left(\tilde{z}^{j}\right)\right\}, \quad \tilde{S}_{x^{j}}=\left\{x: u_{t_{j}}(x)=u_{t_{j}}\left(\tilde{x}^{j}\right)\right\} .
$$

Let moreover $\tilde{z}^{j}=z^{j} / r_{j}, \tilde{x}^{j}=x^{j} / t_{j}, \tilde{z}^{0}=\lim \tilde{z}^{j}$, and $\tilde{x}^{0}=\lim \tilde{x}^{j}$. The existence of the latter are obvious, at least for a subsequence. Next for any fixed ball $B(0, R)$ we have

$$
\begin{aligned}
& \tilde{S}_{z^{j}} \cap B(0, R) \quad \rightarrow \quad\left\{x_{1}=\tilde{z}_{1}^{0}\right\} \cap B(0, R), \\
& \tilde{S}_{x^{j}} \cap B(0, R) \quad \rightarrow \quad\left\{x_{1}=\tilde{x}_{1}^{0}\right\} \cap B(0, R),
\end{aligned}
$$

with $\tilde{z}_{1}^{0}>0$ and $\tilde{x}_{1}^{0}>0$.

An important observation at this stage is that

$$
\left|\nabla u_{r_{j}}\right| \rightarrow \alpha_{1} \quad \text { locally in }\left\{u_{1}>0\right\},
$$

and

$$
\left|\nabla u_{t_{j}}\right| \rightarrow \alpha_{2} \quad \text { locally in }\left\{u_{2}>0\right\} .
$$

Let now $\gamma_{z^{j}}(t)$ be the curve introduced in Lemma 2.7, starting at $z^{j}$ with $t \geq s_{j}:=$ $u\left(z^{j}\right)$. From the same lemma we'll have

$$
|\nabla u|\left(\gamma_{z^{j}}(t)\right) \geq|\nabla u|\left(z^{j}\right) \geq \alpha_{1}-\epsilon_{j},
$$

for $t \geq s_{j}$ and $\epsilon_{j} \rightarrow 0$ as $j \rightarrow \infty$.

Next we estimate the length of $\gamma_{z^{j}}(t)$ from $z^{j}$ to the point $y^{j}$ where $\gamma_{z^{j}}(t)$ hits the set $S_{x^{j}}$. Let $\rho_{j}$ be such that

$$
\gamma_{z^{j}}\left(\rho_{j}\right)=y^{j}, \quad u\left(y^{j}\right)=u\left(x^{j}\right)=\rho_{j} .
$$

Obviously $\rho_{j} \leq C t_{j}$ since $u_{t_{j}}$ is bounded. Now

$$
\int_{s_{j}}^{\rho_{j}}\left\|\gamma_{z^{j}}(t)\right\| d t=\int_{s_{j}}^{\rho_{j}} \frac{d t}{|\nabla u|\left(\gamma_{z^{j}}(t)\right)} \leq \frac{\rho_{j}-s_{j}}{\alpha_{1}-\epsilon_{j}} \leq C t_{j}
$$

where in the first inequality we have used (2.4). From this we conclude that

$$
\left|y^{j}\right| \leq\left|y^{j}-z^{j}\right|+\left|z^{j}\right| \leq C t_{j}+r_{j}<2 C t_{j}
$$

Moreover

$$
|\nabla u|\left(y^{j}\right) \geq \alpha_{1}-\epsilon_{j}
$$

Next (2.5) gives that

$$
y^{j} / t_{j}=: \tilde{y}^{j} \in \tilde{S}_{x^{j}} \cap B(0,2 C) .
$$

But from (2.6) it follows that

$$
\left|\nabla u_{t_{j}}\right|\left(y^{j}\right) \geq \alpha_{1}-\epsilon_{j}
$$

On the other hand by $(2.3),(2.7)$ and Lipschitz continuity of $u$ we have

$$
\left|\nabla u_{t_{j}}\right|(x) \rightarrow \alpha_{2} \leq \alpha_{1}-\epsilon_{0}<\alpha_{1}-\epsilon_{j},
$$

on compact sets of $\left\{u_{2}>0\right\}$ and in particular at $\tilde{y}^{0}:=\lim \tilde{y}^{j}$. This is indeed a contradiction and (2.2) is proved for points on $\partial D_{2}$.

To prove (2.2) for points on $\partial D_{1}$, we replace $u$ by $1-u$ and reverse the role of $x^{j}, z^{j}$ and $t_{j}, r_{j}$ respectively. Then once again the curve $\gamma_{z^{j}}(t)$ hits the set 
$\left\{u(x)=u\left(x^{j}\right)\right\}$ at point $y^{j}$ and $|\nabla u|\left(y^{j}\right) \geq \alpha_{1}$. Now the same holds for the part of the curve $\gamma_{z^{j}}(t)$ which goes from $y^{j}$ to $\partial D_{1}$, and again the length of the curve from $y^{j}$ to $\partial D_{1}$ is approximately $\approx t_{j}$.

To complete the proof let $x^{j} \rightarrow x^{0} \in \partial D_{2}$. Consider a path $\gamma_{x^{j}}(t)$ starting at $x^{j}$ with $t \geq s_{j}:=u\left(x^{j}\right)$. Then as in $(2.4)$

$$
|\nabla u|\left(\gamma_{x^{j}}(t)\right) \geq|\nabla u|\left(\gamma_{x^{j}}\left(s_{j}\right)\right)=|\nabla u|\left(x^{j}\right) .
$$

Now let $t_{0}$ be large enough and set $z_{j}:=\gamma_{x^{j}}\left(t_{0}\right)$. Then, if necessary, by taking $t_{0}$ even larger we may verify that

$$
\frac{1}{2}\left|z^{j}-x^{0}\right| \leq \operatorname{dist}\left(z^{j}, \partial D_{2}\right)
$$

i.e., $z^{j}$ approaches $x^{0}$ non-tangentially. In particular, by (2.8),

$$
\lim \left|\nabla u\left(x^{j}\right)\right| \leq \text { non-tangential limit of }|\nabla u| .
$$

For $x^{j} \rightarrow \partial D_{1}$ one makes a similar but reverse argument.

Lemma 2.8. Retain the hypothesis in Lemma 2.6 and suppose also that $\partial D_{1}$ and $\partial D_{2}$ are $C^{1}$.

Then

$$
|\nabla u(x)| \geq \inf _{y \in E_{D_{2}}}|\nabla u(y)|, \quad \text { for all } x \in D_{2} \backslash \bar{D}_{1} .
$$

This lemma is a consequence of Lemma 2.6.

The next lemma is due to P. Laurence and E. Stredulinsky and has a crucial role in our analysis. Indeed, besides the new technicalities that arise in the case of non-constant boundary gradient data, one of the main ideas in this paper is the use of the next lemma.

Lemma 2.9 (See [LS, Lemma 4.1]). Retain the hypothesis in Lemma 2.8. Suppose moreover $\partial D_{i}(i=1,2)$ contains a line segment $l_{i}$, and that $|\nabla u| \geq c_{0}>0$. Then $|\nabla u|^{-1}$ is convex on $l_{2}$ and it is concave on $l_{1}$.

In the above lemma (and also in the sequel) when we refer to $|\nabla u|$ on the boundary $\partial D_{i}(i=1,2)$ we mean the non-tangential limit from interior of the domain. This exists according to (2.2).

Next we announce the following geometric property of convex domains. The proof is elementary and left to the reader.

Lemma 2.10. Let $D^{*}$ denote the convex hull of a domain $D$, and suppose all points of $\partial D \cap \partial D^{*}$ have unique tangent planes. Then $\partial D^{*}$ is $C^{1}$.

Lemma 2.11. Let $D_{R}=\left\{x_{1}<1\right\} \backslash B\left(x^{R}, R\right)$, where $x^{R}=(-R, 0, \cdots, 0)$. Then for $\alpha, \delta>0$ and small, there exists $u_{R}$ such that

$$
\left\{\begin{array}{cc}
\Delta_{p} u_{R}=0 & \text { in } D_{R}, \\
u_{R}=\alpha+\delta / 2 & \text { on }\left\{x_{1}=1\right\}, \\
u_{R}=0 & \text { on } \partial B\left(x^{R}, R\right), \\
0 \leq u_{R} \leq \alpha+\delta / 2 & \text { in } D_{R}, \\
\left|\nabla u_{R}\right| \leq \alpha+\delta & \text { on } \partial B\left(x^{R}, R\right) .
\end{array}\right.
$$

Proof. The existence follows by taking a sequence of bounded domains

$$
B\left(x^{t}, 1+t\right) \backslash B\left(x^{R}, R\right) \quad\left(x^{t}=(-t, 0, \cdots, 0), t \geq R\right)
$$


converging to $D_{R}$, and considering the corresponding solution $u_{R}^{t}$ in the limit as $t \rightarrow \infty$. Now let $C_{R}=B\left(x^{R}, R+1\right) \backslash B\left(x^{R}, R\right)$ and set

$$
v_{R}=(\alpha+\delta / 2)\left(\frac{\left|x-x^{R}\right|^{(p-n) /(p-1)}-R^{(p-n) /(p-1)}}{(R+1)^{(p-n) /(p-1)}-R^{(p-n) /(p-1)}}\right) \quad(p \neq n),
$$

and

$$
v_{R}=(\alpha+\delta / 2)\left(\frac{\log \left|x-x^{R}\right|-\log R}{\log (R+1)-\log R}\right) \quad(p=n) .
$$

Then $v_{R}$ is $p$-harmonic in $C_{R}$ and $\left|\nabla v_{R}\right| \leq \alpha+\delta$ on $\partial B\left(x^{R}, R\right)$, if $R$ is large enough. Now by the comparison principle $u_{R} \leq v_{R}$ and hence $\left|\nabla u_{R}\right| \leq\left|\nabla v_{R}\right| \leq \alpha+\delta$ on $\partial B\left(x^{R}, R\right)$, if $R$ is large enough.

Lemma 2.12. Let $u_{R}$ be as in the previous lemma and define for $\epsilon>0$

$$
w_{R}=w_{R, \epsilon}=(\alpha+\delta / 2)\left(\frac{u_{R}-\epsilon}{\alpha+\delta / 2-\epsilon}\right)_{+} .
$$

Then the following hold:

1) There exists $\epsilon_{0}, R_{0}$ (positive) such that for $\epsilon \leq \epsilon_{0}$, and $R \geq R_{0}$

$$
\left|\nabla w_{R}\right| \leq \alpha+2 \delta \quad \text { on } \partial\left\{u_{R} \leq \epsilon\right\}=\left\{w_{R}=0\right\} .
$$

2) There exist $\delta_{1}, \delta_{2}>0$ such that

$$
w_{R}>\alpha\left(x_{1}\right)_{+}+\delta_{2} \quad \text { on } \Gamma:=\partial B(0,1) \cap\left\{x_{1}>-\delta_{1}\right\} .
$$

Here we may fix $\delta_{1}$ small and choose

$$
0<\delta_{2}=2 \inf _{\Gamma}\left(u_{R}(x)-\alpha\left(x_{1}\right)_{+}\right) .
$$

The proof is obvious.

\section{Proof of Theorem 1.1 (Bounded And Regular Case)}

Let $K$ be bounded and consider the subclass $\mathcal{E}^{*}$ of $\mathcal{E}=\mathcal{E}(K, a)$ defined as

$$
\mathcal{E}^{*}=\left\{u \in \mathcal{E}: \Omega(u)=\text { convex }, \Delta_{p} u=0 \text { in } \Omega \backslash \bar{K}\right\} .
$$

Let us also associate the support $\Omega=\Omega(u)$ of $u$ to the function itself by the notation $(u, \Omega)$, which we refer to as a supersolution, even though $u$ is a solution to the $p$-Laplacian.

Now we aim to take the intersection of all $\Omega(v)$ with $v \in \mathcal{E}^{*}$. A different way of seeing this is that we actually take $\inf _{v \in \mathcal{E}^{*}} v(x)$. The only difference is that in our way of doing it we work directly with the $p$-capacitary potentials rather than all elements of $\mathcal{E}$ with convex support.

Now if we have two elements $u_{1}, u_{2} \in \mathcal{E}^{*}$ then $\inf \left(u_{1}, u_{2}\right) \in \mathcal{E}$, and it has convex support $\Omega=\Omega_{1} \cap \Omega_{2}$. In particular (by the comparison principle) the $p$-capacitary potential $u$ of $\Omega \backslash \bar{K}$ is in $\mathcal{E}^{*}$; see more details in [HS2].

Hence we define

$$
\Omega=\bigcap_{v \in \mathcal{E}^{*}} \Omega(v)
$$

where $\Omega(v)$ indicates the support of $v$. In order for this to have a meaning we need to show that

$$
\mathcal{E}^{*} \neq \emptyset, \quad \text { and } \quad \operatorname{dist}(\Omega(v), K) \geq \delta_{0}>0
$$


where $v \in \mathcal{E}^{*}$. The latter means that the intersection of all $\Omega(v)$ with $v \in \mathcal{E}^{*}$ does not degenerate to $K$, i.e. $\bar{K} \subset \Omega(u)$. To show this let us take $B(0, R) \supset K$, with $R$ large enough such that the $p$-capacitary potential $u_{R}$ of $B(0, R) \backslash \bar{K}$ has the property $\left|\nabla u_{R}\right| \leq a$ on $|x|=R$. This is possible due to the fact that $a(x) \geq c_{0}>0$, see e.g. [HS2, Section 4]. This implies that $u_{R}$ is a supersolution and that $\mathcal{E}^{*}$ is nonempty.

Next define the function

$$
v_{R}=v_{R, \epsilon}=\left(\frac{u_{R}-1+\epsilon}{\epsilon}\right)_{+}
$$

with $\epsilon$ small enough to ensure

$$
\left|\nabla v_{R}\right|=\frac{\left|\nabla u_{R}\right|}{\epsilon} \geq C_{0} \geq a(x) \quad \text { on }\left\{u_{R}=1-\epsilon\right\}
$$

in the first inequality we have used Hopf's boundary point lemma (see [T]). Now applying Lavrentiev principle (see [Lav or e.g. Step 5 below) we conclude

$$
v_{R} \leq u \leq u_{R}
$$

for $u \in \mathcal{E}$. Consequently

$$
\bar{K} \subset \Omega\left(v_{R}\right) \subset \Omega(u) \subset \Omega\left(u_{R}\right) .
$$

Actually the stronger result (3.1) follows from this.

By the stability argument above we can, therefore, extract a sequence $\left(\Omega_{j}, u_{j}\right)$ of supersolutions with $p$-harmonic $u_{j}$ and such that

$$
u_{j} \geq u_{j+1}, \quad \Omega_{j} \supset \Omega_{j+1}, \quad \Omega=\bigcap \Omega_{j} .
$$

Here $\Omega_{j}=\Omega\left(u_{j}\right)$. It is also obvious that $u=\inf u_{j}$. Indeed, by $C^{\alpha}$ convergence we have that $u_{j}$ converges uniformly to $u$. (See more details in [HS2].)

The main difficulty is to show that

$$
\lim _{\Omega \ni x \rightarrow \partial \Omega}\left(\frac{|\nabla u(x)|}{a(x)}\right)=1 .
$$

Step 1: We claim $\partial \Omega$ is $C^{1}$ :

It suffices to show that at each boundary point there exists a unique tangent plane. Suppose the latter fails. Let $x^{0} \in \partial \Omega$, with two supporting planes $\Pi_{1}, \Pi_{2}$ at $x^{0}$. Then by barrier arguments (Lemma 2.2)

$$
\lim _{\Omega \ni x \rightarrow \partial \Omega \cap \Pi_{1} \cap \Pi_{2} .}|\nabla u(x)|=0 .
$$

Let $\Pi_{3}$ be a third plane supporting $\partial \Omega$ at $x^{0}$ and such that $\Pi_{3} \cap \partial \Omega \subset \Pi_{1} \cap \Pi_{2}$, i.e., $\Pi_{3}$ does not touch any other boundary points of $\Omega$ than those on the intersection of the planes $\Pi_{1}$ and $\Pi_{2}$. Now, move $\Pi_{3}$ towards $\Omega$ such that it cuts off $\Omega$ a small cap $\Sigma$; it may well be a tub-like region. Then a similar argument as that of [HS2, proof of Lemma 3.4] will imply that the $p$-capacitary potential of domain $(\Omega \backslash \Sigma) \backslash K$ is in the class $\mathcal{E}^{*}$. This contradicts the minimality of $\Omega$ and completes the proof.

Step 2: $\lim _{y \rightarrow x}|\nabla u|(y) \geq a(x)$ (non-tangentially) for $x \in \bar{E}_{\Omega}$ and $y \in \Omega$ : By (2.2) and a similar reasoning as that in the proof of Theorem 1.3 we need only to show that

$$
\limsup _{y \rightarrow x}|\nabla u|(y)=a(x), \quad x \in E_{\Omega}, \quad y \in \Omega .
$$

Observe that in the latter we only work with the set $E_{\Omega}$ and not the closure of it. The statement actually follows in the same vein as that in the constant boundary 
condition, i.e. $a(x)=$ constant. There is a minor modification in the proof given in [HS2 Lemma 3.4]. However, the continuity of $a(x)$ is crucial to force through the same technique. We leave the details to the reader.

Step 3: $\limsup _{y \rightarrow x}|\nabla u|(y) \leq a(x)$ for $x \in \partial \Omega$ :

Let us first indicate that by Lemma 2.8 and Step 2 above we have $|\nabla u(x)| \geq$ $\min _{\Omega} a(x) \geq c_{0}$ for all $x \in \Omega \backslash \bar{K}$. Therefore, for large $j$, the operator $L_{u_{j}}$ (where $\left\{u_{j}\right\}$ is the minimizing sequence and $L_{u_{j}}$ is defined in Lemma 2.5) is uniformly elliptic.

Next let $K_{s}=\{x: d(x, K)<s\}$, where $s$ is small enough such that $\bar{K}_{s} \subset \Omega(u)$. Define also $S_{j}=\Omega_{j} \backslash K_{s}$. In particular $S_{j} \supset S_{j+1}$. Now we define the function $v_{j}$ to be a solution of the Dirichlet problem

$$
\begin{cases}L_{u_{j}} v_{j}=0 & \text { in } S_{j}, \\ v_{j}=\left|\nabla u_{j}\right|^{p} & \text { on } \partial K_{s}, \\ v_{j}=(a(x))^{p} & \text { on } \partial \Omega_{j} .\end{cases}
$$

In particular $v_{j} \geq\left|\nabla u_{j}\right|^{p}$ on $\partial S_{j}$. Inside the domain $S_{j}, L_{u_{j}}$ is uniformly elliptic with uniformly $C^{\alpha}$ coefficients. Since also $\left|\nabla u_{j}\right|^{p}$ is a subsolution to the operator $L_{u_{j}}$ (Lemma 2.5) we can apply the comparison principle to obtain $\left|\nabla u_{j}\right|^{p} \leq v_{j}$ in $S_{j}$. As $j \rightarrow \infty$ we can invoke classical results on stability [Lan (cf. also [He]) to conclude that $v_{j} \rightarrow v$ where $v$ is the corresponding solution in $S=\bigcap_{j \geq 1} S_{j}=\Omega \backslash K_{s}$. In particular $|\nabla u|^{p} \leq v(x)$ in $\Omega$ and near the boundary $\partial \Omega$. Since $v(x) \rightarrow a(x)^{p}$ (continuously) as $\Omega \ni x \rightarrow \partial \Omega$ we conclude the desired result.

The convergence of the functions $v_{j}$ to the corresponding solution in the limit domain, is actually not standard. Since both the domains and the operators vary. However, using the uniform ellipticity (since $\left|\nabla u_{j}\right| \geq c_{0}>0$ ) and the convexity of the domains we can apply the same techniques as that in $\mathrm{He}$ to conclude the result.

Step 4: $\lim _{y \rightarrow x}|\nabla u|(y)=a(x)$ (non-tangentially) for $x \in \partial \Omega$ and $y \in \Omega$ :

In view of the previous steps we need only to show that the gradient condition $|\nabla u|=a$ holds on $\partial \Omega \backslash E_{\Omega}$, i.e. on all line segments of $\partial \Omega$. We first notice that by Lemma 2.8 and Step 2

$$
|\nabla u(x)| \geq \inf _{x \in E_{\Omega}}|\nabla u(x)| \geq \min a(x)>0, \quad \text { for } x \in \Omega \backslash K,
$$

so that $\lim _{x \rightarrow \partial \Omega}|\nabla u(x)|>0$. Hence by Lemma 2.9 and the concavity of $1 / a$ the function

$$
g(x)=\frac{1}{|\nabla u|(x)}-\frac{1}{a(x)}
$$

is convex, on all line segments $I \subset \partial \Omega$. Now any point on $\partial \Omega \backslash \bar{E}_{\Omega}$ can be considered as a linear combination of $n$ points on $E_{\Omega}$. Therefore it suffices to consider (maximal) line segments with endpoints on the set $\bar{E}_{\Omega}$. Since by previous steps $g(x)=0$ if $x$ is an endpoint of any of such segments, i.e. $x \in \bar{E}_{\Omega}$, we conclude that the convex function $g$ on $I$ must be zero, and thus the desired result.

Step 5: $\lim _{y \rightarrow x}|\nabla u|(y)=a(x)$ for $x \in \partial \Omega$ and $y \in \Omega$ :

This follows from the previous steps and the continuity of the functions $a(x)$. The latter is crucial for the fulfillment of the proof.

Step 6: The uniqueness now follows by the method of Lavrentiev: Suppose two solutions exist, call them $\left(u_{1}, \Omega_{1}\right),\left(u_{2}, \Omega_{2}\right)$. Suppose also $\Omega_{1} \backslash \Omega_{2}$ is nonempty or the reverse. Define $t_{0}=\sup t$ such that $t<1$ and $\left\{u_{2}\left(t\left(x-x^{0}\right)+x^{0}\right)>\right.$ 
$0\} \supset\left\{u_{1}>0\right\}$, where $x^{0}$ is the given point in the theorem. By comparison principle $u_{2}\left(t_{0}\left(x-x^{0}\right)+x^{0}\right) \geq u_{1}(x)$ in $\left\{u_{1}>0\right\} \backslash t_{0}^{-1} \bar{K}$. Hence

$$
t_{0}\left|\nabla u_{2}\right|\left(t_{0}\left(x-x^{0}\right)+x^{0}\right) \geq\left|\nabla u_{1}\right|(x)
$$

at

$$
y^{0} \in \partial\left(\left\{u_{2}\left(t_{0}\left(x-x^{0}\right)+x^{0}\right)>0\right\} \cap\left\{u_{1}(x)>0\right\}\right) ;
$$

the latter is obviously nonempty since otherwise there is nothing to prove. In particular $t_{0} a\left(t_{0}\left(y^{0}-x^{0}\right)+x^{0}\right) \geq a\left(y^{0}\right)$, with $t_{0}<1$. This contradicts the assumption in the theorem.

\section{Proof of Theorem 1.2 (Bounded and regular case)}

For the interior problem, we consider a similar situation as that in the exterior. Here, however, it has an advantage working with the class $\mathcal{I}$ rather than with a subclass of functions with convex support. The reason is that the support of $\min \left(u_{1}, u_{2}\right)$ is not convex. Even though we may be able to take the convex hull of the support of $\min \left(u_{1}, u_{2}\right)$, it would be hard to show that on the new boundary points the gradient condition is verified.

It will be more direct to consider the class $\mathcal{I}$ itself specially when we want to use barriers of the type constructed in Lemmas 2.12-2.13. So let us first assume $K$ is bounded and then, as in [HS3, take

$$
u=\inf _{v \in \mathcal{I}} v
$$

Observe that by the assumptions of the theorem

$$
\mathcal{I} \neq \emptyset \quad \text { and } \quad 0<c_{0} \leq a(x) \leq C_{0} .
$$

Therefore the infimum function $u$ exists, and $\partial\{u>0\}$ does not degenerate to $\partial K$ (see the beginning of the proof of Theorem 1.1). One also observes that $u$ is continuous $\left(\operatorname{Lip}_{l o c}(K) \cap C^{\alpha}\left(\mathbb{R}^{n}\right)\right)$, and it is the $p$-capacitary potential of the set $\{u>0\}$; see [HS2] for more details.

Now let us consider a minimizing sequence $u_{j}$. Since the minimum of two elements in $I$ is again in $I$, we may consider a decreasing sequence

$$
u_{j} \geq u_{j+1}, \quad \Omega\left(u_{j}\right) \subset \Omega\left(u_{j+1}\right) .
$$

Observe that $\Omega(u)=\{u \equiv 0\}$ in the interior case. Next we take the convex hull $\Omega^{*}$ of $\Omega$. We show that the $p$-capacitary potential $u^{*}$ of $K \backslash \Omega^{*}$ is also in $I$. Since it is also smaller than $u$ we may only work with convex domains and the $p$-capacitary potentials of the ring-shaped region. To verify that claim, we first observe that $\left|\nabla u^{*}\right| \leq|\nabla u| \leq a(x)$ on $\partial \Omega^{*} \cap \Omega$, and in particular on $E_{\Omega^{*}}$.

Now, $\partial \Omega \cap \partial \Omega^{*}$ has a unique tangent plane. Since otherwise we may use the interior barrier argument in Lemma 2.3 to reach a contradiction to the Lipschitz regularity of $u$ near $\partial \Omega^{*}$.

Next Lemma 2.11 applies to conclude that $\partial \Omega^{*}$ is $C^{1}$. Hence Lemma 2.10 in conjunction with the convexity assumption of $a^{-1}$ implies that

$$
g(x)=\frac{1}{|\nabla u|(x)}-\frac{1}{a(x)}
$$

is concave on line segments of $\partial \Omega^{*}$. We also need that $|\nabla u|>0$ on $\partial \Omega^{*}$. This follows by Hopf's Lemma (since $\partial \Omega^{*}$ satisfies the interior, w.r.t. $K \backslash \Omega$, sphere condition). Now realizing that any point of $\partial \Omega^{*} \backslash E_{\Omega^{*}}$ can be put on a line segment 
with endpoints on $E_{\Omega^{*}}$ and since $g \geq 0$ on these endpoints we conclude that $g \geq 0$ also on the rest of the boundary of $\Omega^{*}$. This in particular implies that $u^{*} \in \mathcal{I}$, and that the minimal element $u^{*}$ is such that $\Omega^{*}$ is convex. As in Step 1 in the proof of Theorem 1.1, we conclude that

$$
\left|\nabla u^{*}\right| \leq a(x)
$$

for the minimal element $u^{*}$ of $\mathcal{I}$. Here, again, we need to assure that $\left|\nabla u_{j}\right| \geq c_{0}>0$. By the interior sphere condition, this can be done using Hopf's boundary point lemma $[\mathrm{T}$.

Now we must show that $\left|\nabla u^{*}\right| \geq a$. To this end, Lemmas $2.12-2.13$ are helpful to construct a new smaller element in $\mathcal{I}$ if the gradient $u$ becomes smaller than $a(x)$ at some boundary point.

Let $y \in \partial \Omega^{*}$ and suppose $|\nabla u|(y)<a(y)$. Then we reach a contradiction.

To simplify the geometric picture, we assume $y$ is the origin and the outward normal vector to $\Omega$ at $y$ is the $x_{1}$-axis. Then by Lemma 2.8 the sequence $u_{r_{j}}=$ $u\left(r_{j} x\right) / r_{j}$ converges to $\alpha\left(x_{1}\right)_{+}$. Hence, for $r_{j}$ small enough,

$$
u<\alpha\left(x_{1}\right)_{+}+o\left(r_{j}\right),
$$

inside a ball of center 0 and radius $r_{j}$.

Now using Lemma 2.13, we may take the function $w_{R}$ constructed there in a rescaled form $\tilde{w}=r_{j} w_{R}\left(\frac{x}{r_{j}}\right)$. By part 2) of Lemma 2.12

$$
\tilde{w}>\alpha\left(x_{1}\right)_{+}+r_{j} \delta_{2}>u \quad \text { on } \partial B\left(0, r_{j}\right),
$$

provided $r_{j}$ is small enough.

Let now

$$
v= \begin{cases}\min (u, \tilde{w}) & \text { in } B\left(0, r_{j}\right) \\ u & \text { in } \mathbb{R}^{n} \backslash B\left(0, r_{j}\right) .\end{cases}
$$

Then $v \in \mathcal{I}$, and since $v$ is identically zero in a small neighborhood of the origin we'll have a contradiction to the minimal property of $u$. Therefore $|\nabla u|=a$ on $\partial \Omega$. The proof of Theorem 1.2 is now complete.

\section{Unbounded/IRREGUlar $K$, AND SOME RETROSPECT}

5.1. Unbounded and irregular case. The irregular case of $K$, can be handled easily either by approximation of the set $K$ by smooth domains, or by enlargening the definition of the classes $\mathcal{E}$ and $\mathcal{I}$ to $C^{\alpha}$ functions. Here $\alpha$ depends on the regularity of $\partial K$. Observe that if we already have considered the minimum $u$ in the class $\mathcal{E}^{*}$ or in $\mathcal{I}$ we may replace the set $K$ by a level set $K^{\prime}:=\{u>1 / 2\}$ in the exterior case and $K^{\prime}:=\{u<1 / 2\}$ in the interior case. Since then we just consider the problem over the new domains $K^{\prime}$ in each case. These domains have analytic boundaries by results of John Lewis $[\mathrm{L}$. Obviously any solution for the new problem is also a solution to the original problem with $K$ replaced by $K^{\prime}$, due to the minimal properties of $u$. We leave the obvious detail to the reader.

The unbounded case of Theorems 1.1-1.2 are handled again by approximation. We take $K_{R}:=K \cap B(0, R)$ and solve the problem for $K_{R}$. Then we let $R \rightarrow \infty$, the solutions are monotone increasing and have a limit. So we only need to study the properties of the limit function. First one easily verifies that the limit solution is indeed the $p$-capacitary potential for the corresponding problem, due to the fact that the increasing family $u_{R}$ are locally uniformly $C^{\alpha}$. Actually they also are bounded by construction. 
The core difficulty is the use of Lemma 2.9. Here however, again one applies the same lemma and it works perfectly even though we consider unbounded line segments, i.e., rays. One should notice that the function $g(x)$ introduced in the proofs of the theorems remain bounded on the rays. Once again the details are left to the reader.

5.2. Qualitative analysis. An interesting qualitative analysis in line with the results in [GS Theorem 3.9] can be done here as well. In order not to repeat the same arguments, already done by [GS, and many others for that matter, we only mention what type of qualitative results are to be expected for our problems. We focus on the exterior problem only.

Suppose, in addition, the continuous function $a(x)$ is non-decreasing in direction $e$. Let also $T$ be a supporting plane to $\Omega$ with normal $e$ so that $T^{-}:=\{x: x \cdot e<$ $0\} \supset K$. Then one can show that the set $\Omega \cap T^{-}$, has this property as well. This can be verified, by building this property into the construction of the solution and then using uniqueness for the exterior case. Since the uniqueness fails in general for the interior case we may only construct solutions with this property. But we can't prove this property for an already existing solution in the interior cases.

The method of moving plane is a well known technique for such qualitative analysis; see e.g. GNN], [S]. The technique of Serrin cannot be adopted directly since the boundary $\partial \Omega$ is only known to be $C^{1}$. However, in our construction we may take a sequence of minimizer that already has such a property, and hence the limit domain (function) will have such a property. See [GS, the proof of Theorem 3.9] for details.

Yet another property that might be of interest is the asymptotic behavior of the solution $u_{\lambda}$ for the function $a_{\lambda}(x)=\lambda a(x)$ (or one may consider even more complicated functions, but carefully). Indeed, the same analysis of reflection, moving planes, (see [GS, Theorem 3.9]) will show that the solution will eventually converge to a ball of radius $\infty$ as $\lambda \rightarrow 0$. It would be interesting to analyze the exact quantitative behavior of such solutions in terms of the inner and outer radius, i.e., the radius of the largest ball inside and the radius of the smallest ball outside.

5.3. Further horizon. As already mentioned in the introduction, the free boundary problem discussed in this paper, for $p=2$, has been studied intensively in the past 20 years. Even though the case $1<p<\infty$ seems more realistic in applications, there are today not many results for this most simple case, simple in nonlinear degenerate setting.

The development of the Bernoulli free boundary problem (in the multi-phase, and uniformly elliptic case) has flourished since the pioneering works of Carleman (1918), Friedrich (1934), and A. Beurling (1957) (see the introduction in [GS] for some historical accounts). One of the major contributions, for the existence in a general framework is given in the paper by $\mathrm{H}$. Alt and L. Caffarelli $\mathrm{AC}$, where the authors use minimization of certain cost functionals. It would be interesting, and it is definitely tantalizing, to see a similar development for the case of the $p$-Laplacian.

The more milder method of A. Beurling, developed in [HS2]-[HS3], seems also possible for the general problem, i.e., with no geometric assumptions on data. This was developed by L. Caffarelli in [C1]-C3 for uniformly elliptic operators. The difficulty in this type of approach is the consideration of the function space and the boundary gradient condition. L. Caffarelli overcame this problem by replacing the gradient condition with an asymptotic development for the solution (a better 
version of Lemma 2.4). This also seems to be a possible way to develop the theory for the $p$-Laplacian using viscosity solutions.

Other methods that have in some extent been developed (and they probably are under investigation) are the operator method of A. Acker [AM], and the use of Nash-Moser inverse function theorem by Hamilton $[\mathrm{H}]$. The latter technique has gained some renewed interest in the porous medium equation $[\mathrm{DH}]$.

Finally, let us mention the method of singular perturbation, which also has been in much focus lately. The technique of singular perturbation is much reminiscent of the penalizing technique for the obstacle problem. One replaces the problem with a new one by finding a function $u_{\epsilon}$ which solves, say locally,

$$
\Delta u_{\epsilon}=\beta_{\epsilon}\left(u_{\epsilon}\right) .
$$

Here $\beta_{\epsilon}$ is the absorption term and it vanishes outside the set $D_{\epsilon}:=\left\{0<u_{\epsilon}<\epsilon\right\}$. In particular $u_{\epsilon}$ becomes harmonic outside $\bar{D}_{\epsilon}$. To derive the boundary gradient condition $|\nabla u|(x)=a(x)$ we need to impose certain conditions on $\beta$. One such condition is that

$$
\lim _{\epsilon \rightarrow 0} \beta_{\epsilon}\left(u_{\epsilon}\right)=(n-1) \text {-Hausdorff measure restricted to } \partial\{u>0\} .
$$

This technique has also been developed thoroughly for both elliptic and parabolic problems, see [BCN], [CLW]. However, still in the framework of uniformly elliptic (and parabolic) case.

A recent attempt has been made by the second author, D. Danielli, and A. Petrosyan to generalize the technique of singular perturbation to the case of $p$ Laplacian DPS.

In closing, we would like to mention that the Bernoulli free boundary problem seems yet to surprise us with the contribution of many varieties of beautiful and strong techniques, which are widely used in many other areas of partial differential equations. We thank the reader for his/her time.

\section{REFERENCES}

[A] A. ACKER, On the existence of convex classical solutions for multi-layer free boundary problems with general nonlinear joining conditions. Trans. Amer. Math. Soc. 350 (1998), no. 8, 2981-3020. MR 98k:35209

[AM] A. ACKer AND R. Meyer, A free boundary problem for the p-Laplacian: uniqueness, convexity and successive approximation of solutions, Electronic Journal of Differential Equations, 8 (1995), 1-20. MR 96c:35198

[AC] H.W. Alt and L.A. Caffarelli, Existence and regularity for a minimum problem with free boundary, J. Reine Angew. Math., 325 (1981), 105-144. MR 83a:49011

[BCN] H. Berestycki, L. Caffarelli, L. Nirenberg, Uniform estimates for regularization of free boundary problems. Analysis and partial differential equations, 567-619, Lecture Notes in Pure and Appl. Math., 122, Dekker, New York, 1990. MR 91b:35112

[C1] L.A. CAfFarelli, A Harnack inequality approach to the regularity of free boundaries. I. Lipschitz free boundaries are $C^{1, \alpha}$. Rev. Mat. Iberoamericana 3 (1987), no. 2, 139-162. MR 90d:35306

[C2] L.A. CAfFarelli, A Harnack inequality approach to the regularity of free boundaries. II. Flat free boundaries are Lipschitz. Comm. Pure Appl. Math. 42 (1989), no. 1, 55-78. MR 90b:35246

[C3] L.A. CAFFARELLI, A Harnack inequality approach to the regularity of free boundaries. III. Existence theory, compactness, and dependence on $X$. Ann. Scuola Norm. Sup. Pisa Cl. Sci. (4) 15 (1988), no. 4, 583-602 (1989). MR 91a:35170 
[ClW] L.A. Caffarelli, C. Lederman, N. Wolanski, Uniform estimates and limits for a two phase parabolic singular perturbation problem. Indiana Univ. Math. J. 46 (1997), no. 2, 453-489. MR 98i:35099

[CS] L.A. Caffarelli, J. Spruck, Convexity properties of solutions to some classical variational problems, Comm. Part. Diff. Eq., 7 (1982), 1337-1379. MR 85f:49062

[DH] P. Daskalopollos, R. Hamilton, Regularity of the free boundary for the porous medium equation. J. Amer. Math. Soc. 11 (1998), no. 4, 899-965. MR 99d:35182

[Di] E. DiBenedetto, Degenerate parabolic equations. Universitext. Springer-Verlag, New York, 1993. MR 94h:35130

[DPS] D. Danielli, A. Petrosyan, H. Shahgholian, A singular perturbation problem for the p-Laplacian, with Bernoulli boundary condition. Submitted.

[Do] Dobrowolski M. On quasilinear elliptic equations in domains with conical boundary points, J. Reine Angew. Math 394 (1989), 186-195. MR 90c:35087]

[FR] M. Flucher And M. RumpF, Bernoulli's free boundary problem, qualitative theory and numerical approximation, J. Reine Angew. Math., 486 (1997), 165-204. MR 98i:35214

[GNN] B. Gidas, W. Ni, L. NirenberG, Symmetry of positive solutions of nonlinear elliptic equations in $\mathbb{R}^{n}$. Mathematical analysis and applications, Part A, pp. 369-402, Adv. in Math. Suppl. Stud., 7a, Academic Press, New York-London, 1981. MR 84a:35083

[GT] D. Gilbarg And N. Trudinger, Elliptic partial differential equations of second order, Springer Verlag, New York 1983. MR 86c:35035

[GS] B. Gustafsson, H. Shahgholian, Existence and geometric properties of solutions of a free boundary problem in potential theory. J. Reine Angew. Math. 473 (1996), 137-179. MR 97e:35205

[H] R. Hamilton, The inverse function theorem of Nash and Moser. Bull. Amer. Math. Soc. (N.S.) 7 (1982), no. 1, 65-222. MR 83j:58014

[HKM] J. Heinonen, T. Kilpeläinen, O. Martio, Nonlinear Potential Theory Of Degenerate Elliptic Equations, Oxford University Press, Oxford, 1993. MR 94e:31003

[He] A. Henrot Continuity with respect to the domain for the Laplacian: a survey. Shape design and optimization. Control Cybernet. 23 (1994), no. 3, 427-443. MR 95i:35030

[HS1] A. Henrot and H. Shahgholian, Convexity of free boundaries with Bernoulli type boundary condition, Nonlinear Analysis T.M.A., 28, n 5, 1997, 815-823. MR 97j:35165

[HS2] A. Henrot and H. Shahgholian, Existence of classical solutions to a free boundary problem for the p-Laplace operator: (I) the exterior convex case. J. Reine Angew. Math. 521 (2000), 85-97. MR 2001f:35442

[HS3] A. Henrot and H. Shahgholian, Existence of classical solutions to a free boundary problem for the p-Laplace operator: (II) the interior convex case. Indiana Univ. Math. J. 49 (2000), no. 1, 311-323. MR 2001m:35326

[K] I.N. KroL, The solutions of the equation $D_{x_{i}}\left(D u^{p-2} D_{x_{i}} u\right)=0$ with a singularity at a boundary point, Boundary value problems of mathematical physics, 8. Trudy Mat. Inst. Steklov. 125 (1973), p. 127-139. MR 49:9409

[Lan] N. S. LANDKOF, Foundations of modern potential theory. Translated from the Russian by A. P. Doohovskoy. Die Grundlehren der mathematischen Wissenschaften, Band 180. Springer-Verlag, New York-Heidelberg, 1972. MR 50:2520

[Lav] M. Lavrentiev, Variational Methods, Noordhoff, Groningen (1963).

[L] J.L. Lewis, Capacitary functions in convex rings, Arch. Rational Mech. Anal. 66 (1977), 201-224. MR 57:16638

[LS] P. Laurence, E. Stredulinsky, Existence of regular solutions with convex levels for semilinear elliptic equations with nonmonotone $L^{1}$ nonlinearities. Part (I). Indiana Univ. Math. J. vol. 39 (4) (1990) pp. 1081-1114. MR 92m:35279

[MPS] J. Manfredi, A. Petrosyan, H. Shahgholian, A free boundary problem for the $\infty-$ Laplacian. Calc. Var. (2001).

[PP1] L.E. Payne And G. Philippin, On the conformal capacity problem, Geometry of solutions to Partial Differential Equations, Symposia Mathematica, G. Talenti ed., Academic Press (1989), New York, p. 119-136. MR 91h:35067

[PP2] L.E. Payne And G. Philippin, On gradient maximum principles for quasilinear elliptic equations, Nonlinear Analysis T.M.A., 23, n 3 (1994), p. 387-398. MR 95h:35035

[S] J. SERrin, A symmetry problem in potential theory. Arch. Rational Mech. Anal. 43 (1971), 304-318. MR 48:11545 
[T] P. TOLKSDORF On the Dirichlet problem for quasilinear equations in domains with conical boundary points. Comm. in Part. Diff. Equations, 8(7), 773-817 (1983). MR 85g:35053

[V] A. Vogel, Symmetry and regularity for general regions having a solution to some overdetermined boundary value problems, Atti. Sem. Mat. Fis. Univ. Modena, XL, 1992, p. 443-484. MR 94b:35187

Ecole des Mines and Institut Elie Cartan, UMR CNRS 7502 and INRIA BP 239, 54506 Vandoeuvre-Les-Nancy Cedex, France

E-mail address: henrot@iecn.u-nancy.fr

Department of Mathematics, Royal Institute of Technology, 10044 Stockholm, SweDEN

E-mail address: henriks@math.kth.se 